\title{
Frequency-Hopping/ $M$-ary Frequency-Shift Keying for Wireless Sensor Networks: Noncoherent Detection and Performance
}

\author{
Fucheng Yang ${ }^{\# 1}$, Lie-Liang Yang ${ }^{* 2}$, Huangfu Wei ${ }^{* 3}$, Limin Sun ${ }^{* 4}$ \\ \# School of Electonics and Computer Science \\ University of Southampton, SO17 1BJ, United Kingdom \\ ${ }^{1}$ fyle08@ecs.soton.ac.uk, ${ }^{2} 1$ lyeecs.soton.ac.uk \\ * Institute of Software, Chinese Academy of Sciences \\ Beijing, P. R. China \\ ${ }^{3}$ huangfuwei@is.iscas.ac.cn, ${ }^{4}$ sunlimin@is.iscas.ac.cn
}

\begin{abstract}
In this paper, a novel WSN framework, namely the FH/MFSK WSN, is proposed, which monitors $M$-ary events and conveys signals from local sensors to fusion center with the aid of frequency-hopping $(\mathrm{FH})$ and $M$-ary frequency-shift keying (MFSK) techniques. The source events under observation by local sensors are assumed to have $M$ states. The estimates of local sensors are transmitted to the fusion center using MFSK modulation aided by FH. Channels from local sensors to fusion center are modelled either as additive white Gaussian noise (AWGN) channels or as Rayleigh fading channels. At the fusion center, signals are noncoherently detected based on square-law principles aided by equal gain combining (EGC). In this paper, the detection performance of the FH/MFSK WSN is investigated by simulation approaches. Our studies show that the FH/MFSK constitutes one of the promising schemes for efficient information delivery in WSNs. Reliable detection can be achieved at reasonable SNR levels for detection at local sensors and at fusion center.
\end{abstract}

\section{INTRODUCTION}

Due to the great potential for many applications and also due to the advancement of emerging technologies, wireless sensor networks (WSNs) have drawn intensive research in recent years. In WSNs, signal detection constitutes one of the very important tasks, and a lot of research effort has been made for design of high-efficiency low-complexity detection algorithms [1-10]. Specifically, for binary events monitored, optimum and sub-optimum detection algorithms have been derived under various optimization criteria. Detection schemes found in literature include Neyman-Pearson detection [2,9], Bayes detection $[2,5,9]$, maximum likelihood detection $[2-6$, 9], maximal ratio combining detection and equal gain combining detection [3-6], Chair-Varshney fusion detection [4$6]$, etc. In order to improve spectral efficiency and reduce detection delay, in [11], a multiple-access model has been proposed for transmission of signals from local sensors to fusion center and corresponding fusion detection rules have been studied. Furthermore, in [7], the fusion detection of $M$-ary events has been investigated by merging the fusion detection with channel decoding. Owing to its low-complexity, in WSNs, noncoherent detection is often preferred to the coherent detection, which requires extra complexity and extra resources for channel estimation [12].
In this paper, we propose and investigate a novel WSN framework, which considers noncoherent detection of $M$-ary events. Our proposed WSN has a parallel triple-layer network structure [4]. Its source event observed has $M$ possible states, which are assumed to be conveyed to the local sensors in the form of $M$-ary amplitude-shift keying (MASK) signals disturbed by additive white Gaussian noise (AWGN). After the observation, the local sensors transmit their decisions to the fusion center using $M$-ary frequency-shift keying (MFSK) modulation with the aid of frequency-hopping $(\mathrm{FH})[12,13]$. Therefore, for brevity, the proposed WSN is referred to as the FH/MFSK WSN. In the proposed WSN, the FH technique is introduced for the sake of enhancing the detection reliability by making efficient use of the frequency diversity existing in wireless channels. This frequency diversity turns out to be more significant in performance delivery, when the local sensors are located close to each other, resulting in that their channels to the fusion center become highly correlated. The MFSK is a well-known modulation scheme that is beneficial to using square-law noncoherent detection. Hence, low-complexity fusion detection is attainable. In this contribution, the detection performance of the FH/MFSK WSN is investigated, when the wireless channels from local sensors to fusion center are modelled as AWGN or Rayleigh fading channels. Our analysis and performance results illustrate that the FH/MFSK constitutes one of the promising schemes for efficient information delivery from local sensors to fusion center. It works with $M$-ary events, benefits from the advantages of noncoherent MFSK and FH techniques, and makes efficient use of the diversity existing in both space and frequency domains. Furthermore, it is capable of achieving reliable detection at reasonable SNR levels for the detection at local sensors and at fusion center.

The reminder of this paper is organized as follows. In Section II, we provide the details of the proposed FH/MFSK WSN, where the source event, sensor processing and fusion processing are considered. In Section III, the characteristics of the FH/MFSK WSN is analyzed. Section IV provides performance results and corresponding discussion. Finally, in Section V, our conclusions are derived. 


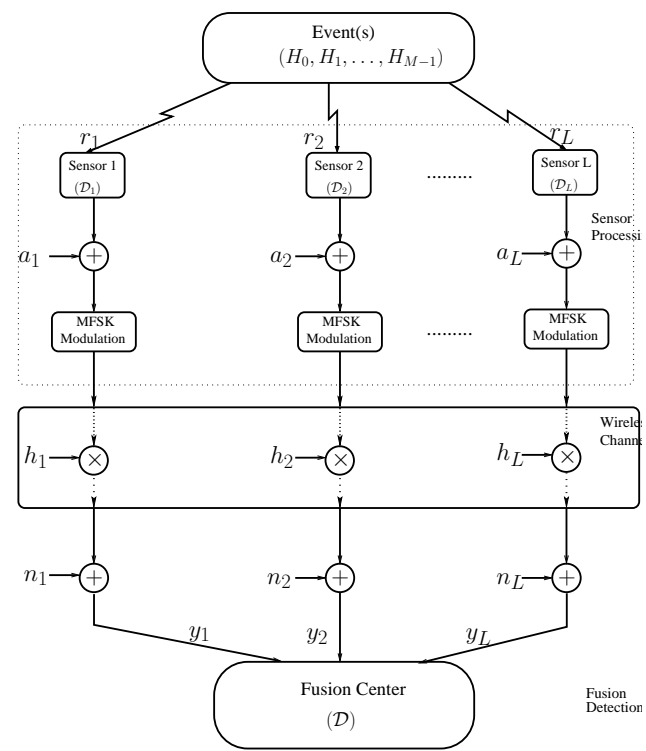

Fig. 1. Triple-layer system model for the WSNs observing an $M$-ary event where information is transmitted to the fusion center based on FH/MFSK scheme.

\section{System Description}

The sensor network considered in this contribution is shown in Fig. 1, which is a typical triple-layer WSN model widely used for research in literature [4-10]. As shown in Fig. 1, the $L$ number of local sensors observe simultaneously an event with $M$ states and then transmit their observed states using the FH/MFSK to the fusion center over wireless channels. The fusion center finally makes a decision on the state of the source event, based on the signals received from the $L$ sensors. Below we describe in detail the components of the WSN considered as well as their operations and corresponding assumptions invoked.

\section{A. Source Event}

The single source event $s$ has $M$ states corresponding to $M$ hypothesises, which are expressed by $H_{0}, H_{1}, \ldots, H_{M-1}$. In this paper, we assume for simplicity that the $M$ hypothesises represent the $M$ amplitudes, $A_{0}, A_{1}, \ldots, A_{M-1}$, obtained by quantizing a continuous event, such as temperature, pressure, etc. Therefore, given at the state $m, m=0, \ldots, M-1$, the event for the local sensors to observe can be represented as

$$
r_{l}=A_{m}+n_{l}, l=1,2, \ldots, L,
$$

where $n_{l}$ is the observation noise

\section{B. Sensor Processing}

When the $l$ th, $l=1, \ldots, L$, sensor obtains an observation in the form of (1) for the source event $s$, it decides the state of $s$ based on the principles of MASK [12]. Let the states estimated by the $L$ sensors are collected to $s=\left[s_{1}, s_{2}, \ldots, s_{L}\right]$, where $s_{l}=m$, if the $l$ th sensor estimates that the source event's amplitude is $A_{m}$. Let us assume that the source event is linearly and uniformly quantized. Then, the observation error probability of the sensors is given by [12]

$$
P_{s e}=2\left(1-\frac{1}{M}\right) Q\left(\sqrt{\frac{6 \log _{2} M}{M^{2}-1} \gamma_{s}}\right)
$$

where $\gamma_{s}$ represents the observation signal-to-noise ratio (SNR) at the sensors, referred to as the sensor SNR for convenience. In practice, the sensor SNR $\gamma_{s}$ is depended on the source event's characteristics, the specific quantization approach used, the sensing method, etc. In (2), $Q(x)$ is the Gaussian $Q$-function defined as $Q(x)=(2 \pi)^{-1 / 2} \int_{x}^{\infty} e^{-t^{2} / 2} d t$. Note that, in our simulations in Section IV, we assume that an erroneous observation leads to one of the $(M-1)$ states other than the correct one with the same probability of $P_{s e} /(M-1)$.

Following the sensing to determine a state of the source event, as shown in Fig. 1, the $L$ sensors transmit their observed states to the fusion center with the aid of the FH/MFSK techniques. The total transmission time is assumed $T_{s}$ seconds, which is referred to as the symbol duration. Let us assume that the WSN system has $M$ orthogonal frequency bands with their center frequencies forming a set $\boldsymbol{F}=\left\{f_{0}, f_{1}, \ldots, f_{M-1}\right\}$. These $M$ frequencies are used for both FH and MFSK modulation, which are implemented as follows. Let $\boldsymbol{a}=$ $\left[a_{1}, a_{2}, \ldots, a_{L}\right]$ be a FH address used by the WSN, where the integer $a_{l} \in\{0,1, \cdots, M-1\}, l=1, \ldots, L$. The purpose of using the FH address is two folds. First, transmitting the information about the source event on different frequency bands is capable of providing frequency-diversity for the detection at fusion center. This becomes even more important, when some sensors are located close to each other, resulting in that their signals received by the fusion center are correlated, if the signals are transmitted on the same frequency band. Second, with the aid of the $\mathrm{FH}$, signals received from the $L$ sensors can be noncoherently combined, which will become explicit in our forthcoming discourse. Based on the FH address $a$ and the estimate $s$, the local sensors first carry out the operation

$$
\begin{aligned}
\boldsymbol{m} & =\left[m_{1}, m_{2}, \cdots, m_{L}\right]=\boldsymbol{s} \oplus \boldsymbol{a} \\
& =\left[s_{1} \oplus a_{1}, s_{2} \oplus a_{2}, \cdots, s_{L} \oplus a_{L}\right]
\end{aligned}
$$

where $\oplus$ represents the addition operation in the Galois field $G F(M)$. Therefore, the value of $m_{l}, l=1, \ldots, L$, is within $[0, M-1]$, suitable for MFSK modulation.

Following the FH operation shown in (3), the components of $\boldsymbol{m}$ are respectively passed to the MFSK modulators of the $L$ sensors, where they are converted to the MFSK frequencies $\boldsymbol{F}_{m}=\left[f_{m_{1}}, f_{m_{2}}, \ldots, f_{m_{L}}\right]$, where $f_{m_{l}} \in \boldsymbol{F}$. Finally, the MFSK modulated signals of the $L$ sensors are transmitted one-by-one to the fusion center in a time-division fashion using $L$ time-slots of duration $T_{h}$, where $T_{h}=T_{s} / L$. Specifically, the signal transmitted by the $l$ th sensor during the $i T_{s}<t \leq(i+1) T_{s}$ can be expressed in complex form as

$$
\begin{aligned}
s_{l}(t)= & \sqrt{P} \psi_{T_{h}}\left(t-i T_{s}-[l-1] T_{h}\right) \\
& \times \exp \left(j 2 \pi\left[f_{c}+f_{m_{l}}\right] t+\phi_{l}\right), l=1, \ldots, L
\end{aligned}
$$

where $P$ denotes the transmission power, which is assumed the same for all the $L$ sensors, $f_{c}$ is the main carrier frequency and $\phi_{l}$ is the initial phase introduced by carrier modulation. In 
(4), $\psi_{T_{h}}(t)$ is the pulse-shaped signalling waveform, which is defined over the interval $\left[0, T_{h}\right)$ and satisfies $\int_{0}^{T_{h}} \psi^{2}(t) d t=$ $T_{h}$.

Assuming that the signal $s_{l}(t), l=1, \ldots, L$, is transmitted over flat Rayleigh fading channels, at the fusion center, the received signal during $i T_{s}<t \leq(i+1) T_{s}$ can be expressed as

$$
\begin{aligned}
r_{l}(t)= & h_{l} s_{l}(t)+n_{l}(t) \\
= & \sqrt{P} h_{l} \psi_{T_{h}}\left(t-i T_{s}-[l-1] T_{h}\right) \\
& \times \exp \left(j 2 \pi\left[f_{c}+f_{m_{l}}\right] t+\phi_{l}\right)+n(t), \\
& \quad l=1, \ldots, L,
\end{aligned}
$$

where $h_{l}=\alpha_{l} \exp \left(j \theta_{l}\right)$ denotes the channel gain with respect to the $i$ th symbol and the $l$ th sensor, which is assumed constant over one symbol-duration. Furthermore, when Gaussian channels are assumed, we have $\alpha_{l}=1$. In (5), $n(t)$ is the Gaussian noise process presenting at the fusion center, which has zero mean and single-sided power-spectral density (PSD) of $N_{0}$ per dimension.

\section{Fusion Processing}

When the fusion center receives the signals in the form of (5), the source event's state is estimated using noncoherent detection approach detailed as follows.

First, corresponding to each of the $L$ sensors, $M$ decision variables can be formed as

$$
\begin{aligned}
R_{m l}= & \mid\left(\sqrt{\Omega P} T_{h}\right)^{-1} \int_{i T_{s}+(l-1) T_{h}}^{i T_{s}+l T_{h}} r_{l}(t) \psi_{T_{h}}^{*}\left(t-i T_{s}-[l-1] T_{h}\right) \\
& \times\left.\exp \left(-j 2 \pi\left[f_{c}+f_{m}\right] t\right) d t\right|^{2}
\end{aligned}
$$

where $m=0,1, \ldots, M-1$ and $l=1,2, \ldots, L$, and $\Omega=E\left[\left|h_{l}\right|^{2}\right]$ denotes the average channel power. Since it has been assumed that the $M$ number of frequency bands invoked are orthogonal to each other, hence there is no interference between any two frequency bands. Consequently, upon substituting (5) into (6) and absorbing the carrier phase $\phi_{l}$ into $h_{l}$, we obtain

$$
\begin{gathered}
R_{m l}=\left|\frac{\mu_{m m_{l}} h_{l}}{\sqrt{\Omega}}+N_{m l}\right|^{2}, m=0,1, \ldots, M-1 \\
l=1,2, \ldots, L
\end{gathered}
$$

where, by definition, $\mu_{m m}=1$, while $\mu_{m m_{l}}=0$, if $m \neq m_{l}$. In (7), $N_{m l}$ is a complex Gaussian noise sample collected from the $m$ th frequency band over the $l$ th time-slot, which is given by

$$
\begin{aligned}
N_{m l}=\left(\sqrt{\Omega P} T_{h}\right)^{-1} & \int_{i T_{s}+(l-1) T_{h}}^{i T_{s}+l T_{h}} n(t) \psi_{T_{h}}^{*}\left(t-i T_{s}-[l-1] T_{h}\right) \\
& \times \exp \left(-j 2 \pi\left[f_{c}+f_{m}\right] t\right) d t
\end{aligned}
$$

which has mean zero and a variance of $L N_{0} /\left(\Omega E_{s}\right)=L / \bar{\gamma}_{s}$, where $E_{s}=P T_{s}$ represents the total energy for transmitting one $M$-ary source symbol with each sensor's transmitted energy per symbol being $E_{h}=E_{s} / L, \bar{\gamma}_{s}=\Omega E_{s} / N_{0}$ denotes the average SNR per symbol.
Having obtained $R_{m l}$ for $m=0,1, \ldots, M-1$ and $l=$ $1,2, \ldots, L$, the fusion center removes the $\mathrm{FH}$ imposed by the local sensors by carrying out the operation

$$
Z_{\left(m \ominus a_{l}\right) l}=R_{m l}, m=0,1, \ldots, M-1 ; l=1,2, \ldots, L
$$

where $\ominus$ denotes the subtraction operation in the Galois field $G F(M)$. The operation in (9) means that the element indexed by $m$ is changed to the one indexed by $m^{\prime}=m \ominus a_{l}$.

Finally, the $M$ decision variables for detection of the source event's state can be formed in EGC principles [13] as

$$
\begin{gathered}
Z_{m^{\prime}}=\sum_{l=1}^{L} Z_{m^{\prime} l}=\sum_{l=1}^{L} R_{\left(m^{\prime} \oplus a_{l}\right) l} \\
m^{\prime}=0,1, \ldots, M-1
\end{gathered}
$$

Then, the largest of $\left\{Z_{0}, Z_{1}, \cdots, Z_{M-1}\right\}$ is selected and mapped to an integer in the range $[0, M-1]$, which represents the estimate to the state of the source event observed by the $L$ local sensors.

\section{AnAlysis of CharaCteristics}

First, the fusion detection scheme described in Section II-C is a noncoherent detection scheme, which does not estimate both the carrier phases and the channels associated with the $L$ local sensors. Hence, the FH/MFSK WSN is capable of achieving low-complexity detection. Second, as shown in (10), noncoherent EGC diversity combining scheme is employed, which is optimum for noncoherent detection. Third, in the proposed FH/MFSK WSN, the introduction of FH can improve the diversity gain achievable. As shown in (10), the FH operation makes the combined signals at the fusion center become more uncorrelated, in addition to the uncorrelation resulted from the spatial separation of the local sensors. The FH operation turns out to become more important, if the $L$ local sensors are located close to each other in space. In this case, signals transmitted by the $L$ sensors may be highly correlated in space and space diversity cannot be guaranteed. Additionally, owing to the employment of noncoherent MFSK and FH, the FH/MFSK WSN can benefit from the embedded advantages of the noncoherent MFSK and FH techniques [12, 13].

In the FH/MFSK WSN, the final achievable detection performance is jointly determined by the detection performance of the $L$ local sensors and that of the fusion center. If the detection performance of the $L$ local sensors is poor, then, the overall achievable detection performance will be probably poor, even when the detection at the fusion center is very reliable. Similarly, the overall achievable detection performance will become worse, if the detection at the fusion center becomes less reliable. Hence, when considering the optimization, the fusion detection and the local sensors' detection need to be jointly optimized. Note that, the optimization issue is beyond the scope of this contribution, which, however, constitutes one of our future research topics in the context of the FH/MFSK WSN.

Let us below provide a range of simulation results, in order to characterize the achievable performance of the proposed WSN. 


\section{Simulation Results}

In this section, the simulation results for the error performance of our FH/MFSK WSN are depicted and analyzed. Specifically, we consider the bit error rate (BER) performance for the sake of unifying the WSN with the conventional onehop communication schemes. In this case, the number of bits per symbol is obtained as $b=\log _{2} M$, and natural mapping from binary to $M$-ary is assumed. In this section, the BER performance of the FH/MFSK WSN is investigated, when assuming that signals observed by the local sensors are only disturbed by Gaussian noise, while the channels from the local sensors to the fusion center are either Gaussian or Rayleigh fading channels.

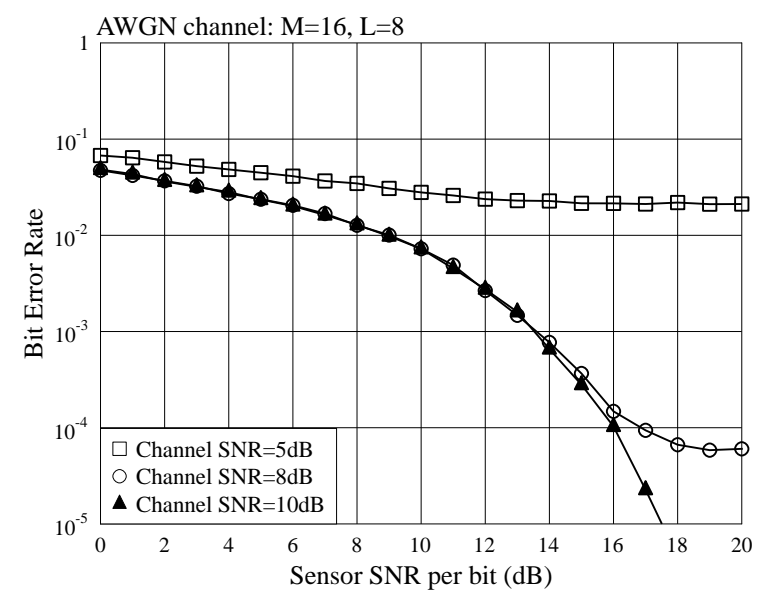

Fig. 2. BER versus sensors SNR per bit performance of the FH/MFSK WSN, when communicating over AWGN channels.

Fig. 2 illustrates the BER performance of the FH/MFSK WSNs, when communicating over AWGN channels. In our simulations, we assumed that $M=16$ frequency bands were used for FH and MFSK, and the WSN used $L=8$ local sensors. From the results, we can explicitly observe that both the local sensors' SNR and the fusion center's SNR have strong impact on the overall detection performance of the FH/MFSK WSN. As shown in Fig. 2, when the channel SNR is sufficiently high, such as $10 \mathrm{~dB}$ per bit, the BER decreases without observing error-floor, as the sensor SNR increases. However, when the channel SNR is relatively low, such as 5 or $8 \mathrm{~dB}$ per bit, then, BER error-floors present, when the sensor SNR increases to a certain level. Furthermore, as seen in Fig. 2, when the channel SNR is $5 \mathrm{~dB}$ per bit, the WSN cannot work properly, even when the channel SNR is very high.

In Fig. 3, we illustrate the BER performance of the FH/MFSK WSNs with respect to various sensor SNR values, when communicating over either AWGN (Fig. 3(a)) or Rayleigh fading (Fig. 3(b)) channels. In our simulations, we assumed that the WSNs employed $L=8$ local sensors and the source event had $M=16$ states. As shown in Fig. 3, when the sensor SNR is not sufficiently high, error-floors appear, when increasing the channel SNR. The reason for this phenomenon is that, when the sensor SNR is relatively low but the channel

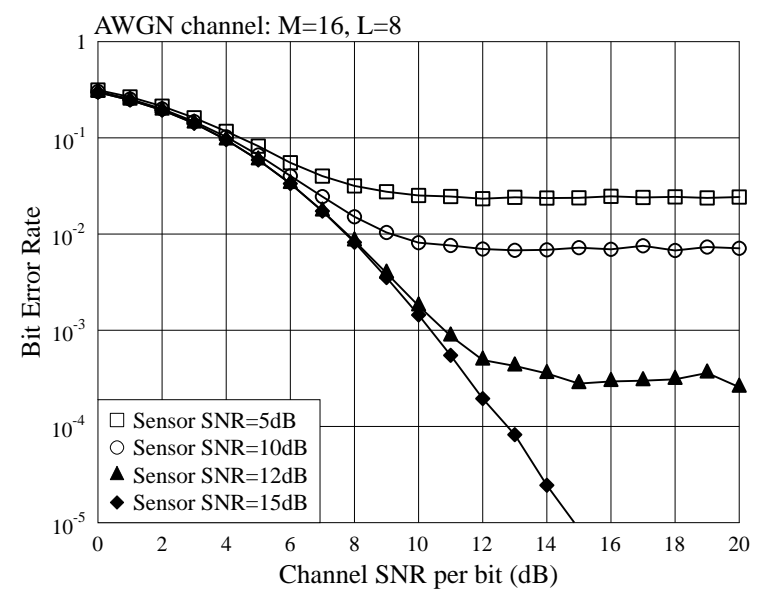

(a) AWGN channel

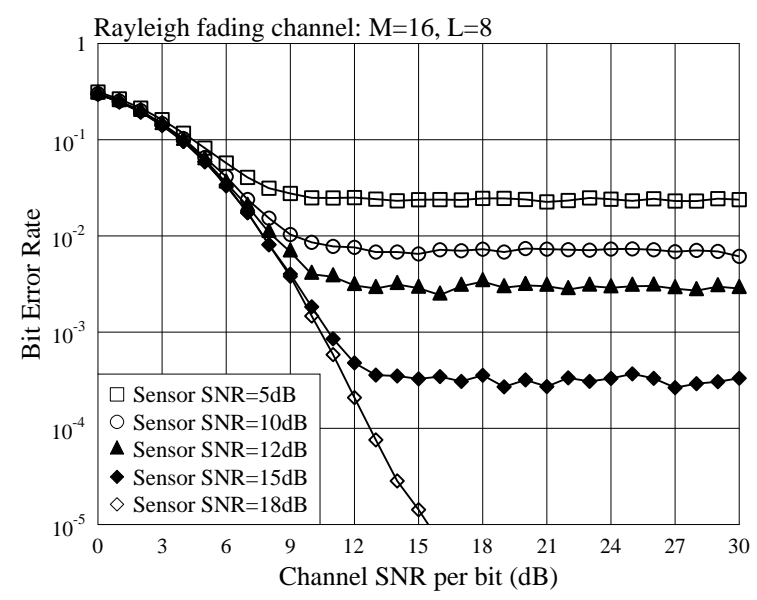

(b) Rayleigh fading channel

Fig. 3. BER versus channel SNR per bit performance of the FH/MFSK WSN, when the WSN employs $L=8$ local sensors and $M=16$ frequency bands for FH and MFSK modulation.

SNR is sufficiently high, the BER performance of the WSNs is dominated by the sensor SNR, which does not decreases with the channel SNR, once it reaches a certain level. By contrast, as seen in Fig. 3, if the sensor SNR is sufficiently high, such as $15 \mathrm{~dB}$ for AWGN channels and $18 \mathrm{~dB}$ for Rayleigh fading channels, the BER performance of the WSNs improves, as the channel SNR increases. There are no error-floors observed in the BER range of interest.

Fig. 4 depicts the BER performance of the FH/MFSK WSNs employing noncoherent detection, when operated over either AWGN (Fig. 4(a)) or Rayleigh fading (Fig. 4(b)) channels. The main objective is to investigate the impact of the number of local sensors on the achievable BER performance of the FH/MFSK WSNs. The cases of $M=16$ and $M=32$ were considered. In our studies, the sensor SNR was assumed to be $20 \mathrm{~dB}$ for both AWGN and Rayleigh fading channels. Explicitly, the results show that the BER performance of the FH/MFSK WSN improves, as the number of local sensors employed by the WSN increases. However, the gain of perfor- 


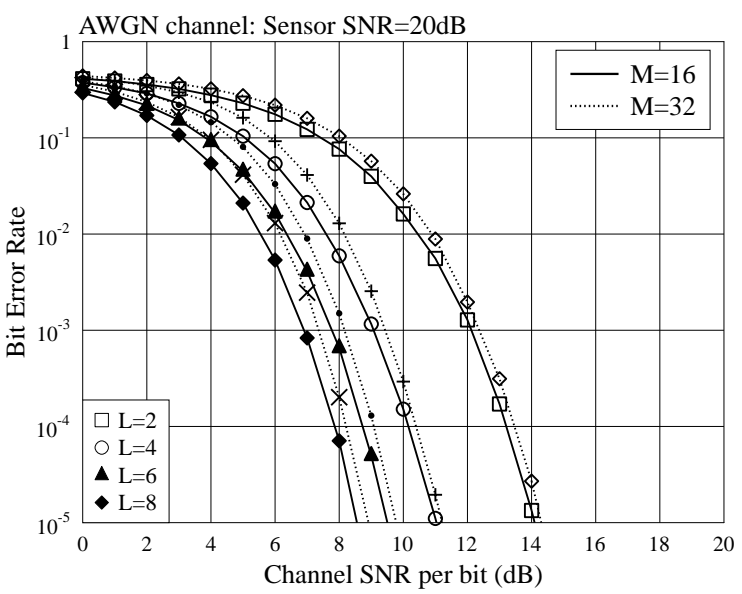

(a) AWGN channel

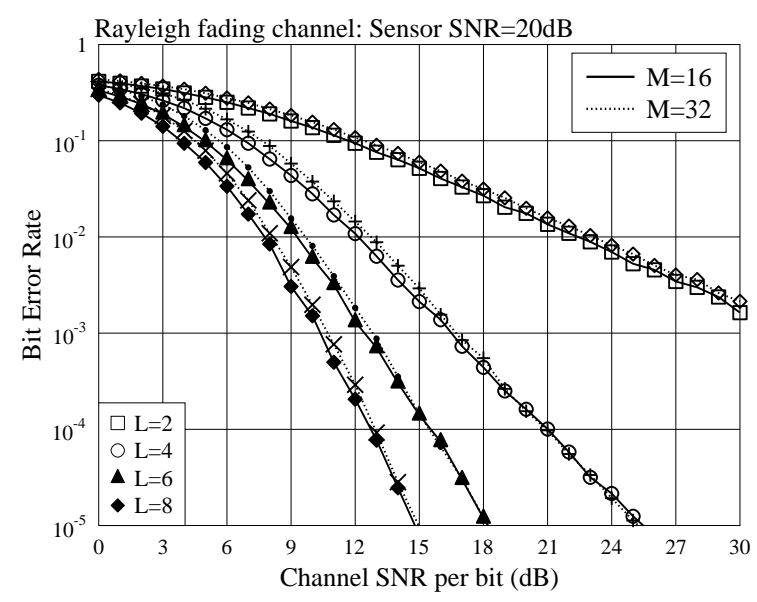

(b) Rayleigh fading channel

Fig. 4. BER versus channel SNR per bit performance of the FH/MFSK WSN, when the WSN employs various number of local sensors and $M=16$ or 32 frequency bands for FH and MFSK modulation.

mance improvement becomes less and less, as the number of local sensors increases. From Fig. 3(b) as well as Fig. 4(b), we can see that, if the sensor SNR is sufficiently high, the WSN with $L=8$ local sensors is capable of attaining a reasonable detection performance, owing to the diversity obtained jointly from the frequency and space domains. Furthermore, as the results in Fig. 4 show, for a given $L$ value, the error performance becomes slightly worse, as the value of $M$ changes from $M=16$ to $M=32$.

Note again that, the BER performance of the WSN is jointly determined by the detection performance of the local sensors and that of the fusion center. In the WSN considered in this contribution, the signals detected by the local sensors are MASK signals, the error performance of which becomes worse with the increase of the value of $M$ [12]. As shown in [12], the BER versus SNR per bit performance of MFSK signals improves with the increase of the value of $M$, when communicating over AWGN channels. It deteriorates, as the value of $M$ increase, when operated in Rayleigh fading channels. Hence, the final achievable BER performance of the WSN is jointly determined by the MASK signals detected at the local sensors and the FH/MFSK signals detected at the fusion center.

\section{CONCLUSION}

A WSN framework has been proposed, which monitors a $M$-ary event whose states are conveyed to the fusion center with the aid of an FH/MFSK scheme. The FH technique has been introduced to enhance the diversity gain, in case that the local sensors are located close to each other, resulting in that their channels to the fusion center are correlated. The MFSK modulation scheme is employed in favour of noncoherent detection for implementing low-complexity detection. The error performance of the FH/MFSK WSN has been investigated, when the channels from sensors to fusion center are AWGN or Rayleigh fading channels. Our studies show that the proposed WSN is capable of achieving promising detection performance for reasonable values of the sensor SNR and channel SNR. However, the achievable detection performance of the FH/MFSK WSN is jointly determined by the local sensors' detection and the fusion center's detection. Hence, when given the total power or energy of the WSN, the local sensor detection strategy and the fusion center detection strategy should be jointly optimized. This constitutes one of our future research topics in the context of the FH/MFSK WSNs.

\section{REFERENCES}

[1] A. Swami, Q. Zhao, Y.-W. Hong, and L. Tong, Wireless Sensor Networks: Signal Processing and Communications Perspectives. Chichester, England: John Wiley \& Sons, 2007.

[2] R. Viswanathan and P. Varshney, "Distributed detection with multiple sensors: Part i-Fundamentals," Proceedings of the IEEE, vol. 85, no. 1, pp. 54 - 63, January 1997.

[3] B. Chen, R. Jiang, T. Kasetkasem, and P. Varshney, "Channel aware decision in wireless sensor networks," IEEE Transactions on Signal Processing, vol. 52, no. 12, pp. 3454 - 3458, December 2004.

[4] R. Niu, B. Chen, and P. Varshney, "Fusion of decisions transmitted over Rayleigh fading channels in wireless sensor networks," IEEE Transactions on Signal Processing, vol. 54, no. 3, pp. 1018 - 1027, March 2006.

[5] B. Chen, L. Tong, and P. Varshney, "Channel-aware distributed detection in wireless sensor networks," IEEE Signal Processing Magazine, no. 3, pp. 16 - 26, July 2006.

[6] Y. Lin, B. Chen, and P. Varshney, "Decision fusion rules in multihop wireless sensor networks," IEEE Transactions on Aerospace and Electronic Systems, vol. 41, no. 2, pp. 475 - 487, April 2005.

[7] T.-Y. Wang, Y. Han, B. Chen, and P. Vaeshney, "A combined decision fusion and channel coding scheme for distributed fault-tolerant classification in wireless sensor networks," IEEE Transactions on Wireless Communications, vol. 5, no. 7, pp. 1695 - 1705, July 2006.

[8] R. Jiang and B. Chen, "Fusion of censored decisions in wireless sensor networks," IEEE Transactions on Wireless Communications, vol. 4, no. 6, pp. 2668 - 2673, November 2005.

[9] S. Aldosari and J. Moura, "Detection in sensor networks: the saddlepoint approximation," IEEE Transactions on Signal Processing, vol. 55, no. 1, pp. 327 - 340, January 2007.

[10] V. Veeravalli and J.-F. Chamberland, "Detection in sensor networks," in Wireless Sensor Networks: Signal Processing and Communications Perspectives, Y.-W. H. A. Swami, Q. Zhao and L. Tong, Eds. John Wiley \& Sons, 2007, pp. $119-148$.

[11] W. Li and H. Dai, "Distributed detection in wireless sensor networks using a multiple access channel," IEEE Transactions on Signal Processing, vol. 55, no. 3, pp. 822 - 833, March 2007.

[12] J. G. Proakis, Digital Communications, 3rd ed. McGraw Hill, 1995.

[13] L.-L. Yang, Multicarrier Communications. Chichester, United Kingdom: John Wiley, 2009. 